\title{
Feedback Stabilization and Lyapunov Functions
}

\author{
F. H. Clarke \\ Institut Girard Desargues (Bât 101) \\ Université Claude Bernard Lyon I (La Doua) \\ 69622 Villeurbanne \\ France \\ Yu. S. Ledyaev \\ Steklov Institute of Mathematics \\ Moscow 117966, Russia \\ L. Rifford \\ Institut Girard Desargues (Bât 101) \\ Université Claude Bernard Lyon I (La Doua) \\ 69622 Villeurbanne \\ France \\ R. J. Stern \\ Department of Mathematics and Statistics \\ Concordia University \\ Montreal, Quebec H4B 1R6, Canada
}

December 17, 1998

\begin{abstract}
Given a locally defined, nondifferentiable but Lipschitz Lyapunov function, we construct a (discontinuous) feedback law which stabilizes the underlying system to any given tolerance. A further result shows that suitable Lyapunov functions of this type exist under mild assumptions. We also establish a robustness property of the feedback relative to measurement error commensurate with the sampling rate of the control implementation scheme.
\end{abstract}

\footnotetext{
${ }^{0}$ Key words:Asymptotic stabilizability, discontinuous feedback law, system sampling, locally Lipschitz Lyapunov function, nonsmooth analysis, robustness

${ }^{0}$ Mathematical Subject Classification: 93D05, 93D20, 34D20, 26B05
} 


\section{Introduction}

Consider a standard control system of the form

$$
\dot{x}(t)=f(x(t), u(t)) \quad \text { a.e., } u(t) \in \mathcal{U},
$$

and let $V$ be a Lyapunov function for the system : We have $V(x) \geq 0, V(x)=$ 0 iff $x=0, V(x) \rightarrow \infty$ as $x \rightarrow \infty$, and (for some function $W$ ) the Infinitesimal Decrease Condition

$$
\min _{u \in \mathcal{U}}\langle\nabla V(x), f(x, u)\rangle \leq-W(x)<0, x \neq 0 .
$$

It is well-known (but true) that the existence of $V$ implies (open-loop) Asymptotic Controllablity to the origin :

for every $\alpha \in \mathbb{R}^{n}$, there is a control $u(t)$ such that the solution $x(\cdot)$ of $(1)$ with initial condition $x(0)=\alpha$ satisfies $x(t) \rightarrow 0$ as $t \rightarrow \infty$ (and in addition, convergence to zero takes place in a certain uniform and stable manner that we will not dwell upon here). A related and important goal in many situations is to produce a state feedback $k(\cdot): \mathbb{R}^{n} \rightarrow \mathcal{U}$ which stabilizes the system; i.e., such that the system $\dot{x}=f(x, k(x))$ is globally asymptotically stable. This article explores the question of how to define such a feedback law through the use of a given Lyapunov function $V$.

The ideal case, a well-known heuristic useful for motivational purposes, is the one in which we can find a continuous function $k(x)$ that selects a value of $u \in \mathcal{U}$ attaining (or almost) the minimum in (2) :

$$
\langle\nabla V(x), f(x, k(x))\rangle \leq-W(x) \quad \forall x \neq 0 .
$$

Then any solution of $\dot{x}=f(x, k(x))$ is such that

$$
\frac{d}{d t} V(x(t))=\langle\nabla V(x(t)), \dot{x}(t)\rangle \leq-W(x)<0,
$$

a monotonicity conclusion that, together with the growth property of $V$, assures that $x(t) \rightarrow 0$ as $t \rightarrow \infty$.

There are two fundamental difficulties with this ideal picture, and both concern regularity issues. The first is that a differentiable Lyapunov function may not exist, and the second is that even when a smooth $V$ exists, the continuous selection $k(\cdot)$ does not generally exist. If we have recourse to a discontinuous feedback $k(\cdot)$, then the issue arises of how to interpret the discontinuous differential equation $\dot{x}=f(x, k(x))$.

The primary goal of this article is to give a general answer to the problem of defining a (discontinuous) stabilizing feedback based upon a given (nondifferentiable) Lyapunov function, one for which infinitesimal decrease is known to hold only on a restricted set. The construction is described in section 1 , while section 2 establishes that under mild conditions, a Lyapunov function of the type required in the previous section always exists. In the final section, we 
address the issue of robustness of the feedback with respect to measurement error and small perturbations of the dynamics, a particularly important issue when discontinuity is present. Some works and general references related to the results of this article include $[2,3,8,11,12,13,15,20,22,23,25,29,30]$. We proceed now to situate our result with respect to the literature.

The possible nonexistence of continuous stabilizing feedback was brought to light in the seminal work of Sontag and Sussmann [28] and of Brockett [4]. The latter who developed a necessary condition for continuous stabilizability and adduced the following example, the "non-holonomic integrator" :

$$
\left\{\begin{array}{l}
\dot{x}_{1}=u_{1} \\
\dot{x}_{2}=u_{2} \\
\dot{x}_{3}=x_{1} u_{2}-x_{2} u_{1}
\end{array} \quad\left(u_{1}, u_{2}\right) \in \bar{B}=: \mathcal{U}\right.
$$

This system is globally asymptotically controllable yet fails to admit a continuous stabilizing feedback (by Brockett's condition). In considering the use of discontinuous feedback laws $k(\cdot)$, one could have recourse to the Filippov solution concept [14] : $x$ is a solution of $\dot{x}=f(x, k(x))=: g(x)$ provided that we have

$$
\dot{x} \in \bigcap_{\substack{\delta>0 \\ \operatorname{meas}(\Omega)=0}} \operatorname{clco}(g([x+\delta B] \backslash \Omega)) .
$$

However, as shown by Ryan [24] and by Coron [11], Brockett's condition continues to hold for this solution concept, so that the nonholonomic integrator (for example) cannot be stabilized by a discontinuous feedback in the Filippov sense.

In [6] it was shown that any globally asymptotically controllable system is stabilizable by a (possibly discontinuous) feedback if the trajectory $x(\cdot)$ associated to the feedback is defined in a natural way that involves discretizing the control law (closed-loop system sampling) in a manner similar to [19]. We proceed now to describe this concept, which is the one used in this article.

Let $\pi=\left\{t_{i}\right\}_{i \geq 0}$ be a partition of $[0, \infty)$, by which we mean a countable, strictly increasing sequence $t_{i}$ with $t_{0}=0$ such that $t_{i} \rightarrow \infty$ as $i \rightarrow \infty$. The diameter of $\pi$, denoted $\operatorname{diam}(\pi)$, is defined as $\sup _{i \geq 0}\left(t_{i+1}-t_{i}\right)$. Given an initial condition $x_{0}$, the $\pi$-trajectory $x(\cdot)$ corresponding to $\pi$ and an arbitrary feedback law $k: \mathbb{R}^{n} \rightarrow \mathcal{U}$ is defined in a step-by-step fashion as follows. Between $t_{0}$ and $t_{1}, x$ is a classical solution of the differential equation

$$
\dot{x}(t)=f\left(x(t), k\left(x_{0}\right)\right), \quad x(0)=x_{0}, \quad t_{0} \leq t \leq t_{1} .
$$

(Of course in general we do not have uniqueness of the solution, nor is there necessarily even one solution, although the latter will be ruled out by the feedback constructed in section 1, which will preclude blow up of the solution in finite time.) We then set $x_{1}:=x\left(t_{1}\right)$ and restart the system with control value $k\left(x_{1}\right)$ :

$$
\dot{x}(t)=f\left(x(t), k\left(x_{1}\right)\right), \quad x\left(t_{1}\right)=x_{1}, \quad t_{1} \leq t \leq t_{2},
$$


and so on in this fashion. The resulting trajectory $x$ is a physically meaningful one that corresponds to a natural sampling procedure and piecewise constant controls; the smaller $\operatorname{diam}(\pi)$, the greater the sampling rate. Since our results are couched in term of $\pi$-trajectories, the issue of defining a solution concept for discontinuous differential equations is effectively sidestepped. Our approach will lead to precise estimates of how small the step $\operatorname{size} \operatorname{diam}(\pi)$ must be for a prescribed stabilization tolerance to ensue, and of the resulting stabilization time, in terms of the given data.

The next major point to address concerns the nonsmoothness of the Lyapunov function $V$. An early and important result of Artstein [1] implies in particular that the nonholonomic integrator fails to admit a smooth $V$ (see [7] for related results). It has been shown by Sontag [25], however, that globally asymptotically controllable systems always admit a continuous Lyapunov function $V$ satisfying the following nonsmooth version of the infinitesimal decrease condition :

$$
\inf _{u \in \mathcal{U}} D V(x ; f(x, u)) \leq-W(x)<0, x \neq 0,
$$

where the lower Dini derivate $D V$ is defined by

$$
D V(x ; v):=\liminf _{\substack{t \downarrow 0 \\ v^{\prime} \rightarrow v}} \frac{V\left(x+t v^{\prime}\right)-V(x)}{t} .
$$

Among the several important ways in which the theory of nonsmooth analysis intervenes in this article is that of asserting the equivalence to (3) of another, and for our purposes more useful, form of the infinitesimal decrease condition :

$$
\inf _{v \in \operatorname{cof}(x, \mathcal{U})}\langle v, \zeta\rangle \leq-W(x)<0 \quad \forall x \neq 0, \forall \zeta \in \partial_{P} V(x) .
$$

Here $\partial_{P} V(x)$ refers to the proximal subdifferential of $V$ at $x$ (which may very well be empty); $\zeta$ belongs to $\partial_{P} V(x)$ iff there exists $\sigma$ and $\eta>0$ such that

$$
V(y)-V(x)+\sigma\|y-x\|^{2} \geq\langle\zeta, y-x\rangle \quad \forall y \in B(x, \eta) .
$$

The equivalence of (3) and (5) is a consequence of Subbotin's Theorem (see for example [9], our principal source for the theory of nonsmooth analysis).

The essential reason for which proximal calculus is well-suited to our approach is because of its relation to metric projection onto sets, upon which is based the "proximal aiming" method that we employ. The crux is this : when $x\left(t_{i}\right)=x$ lies outside a level set $S=S(c):=\{V \leq c\}$ and admits closest point (or projection) $s$ in $S$, then $x-s$ is a "proximal normal" to $S$ at $s$, and for some $\lambda>0$ we have $\lambda(x-s) \in \partial_{P} V(x)$. Then (5) can be invoked at $s$ to find a suitable value of the control $u$ which moves the state toward $S$, in the sense that the Euclidian distance $d_{S}$ decreases at a certain positive rate $\Delta$ :

$$
d_{S}(x(t))-d_{S}\left(x\left(t_{i}\right)\right) \leq-\Delta\left(t-t_{i}\right), \quad \forall t_{i} \leq t \leq t_{i+1}
$$


provided $x\left(t_{i}\right)$ is close enough to $S$ to start with, and provided $\operatorname{diam}(\pi)$ is small enough. A sequence of such feedbacks is amalgamated in the first section to produce the stabilizing feedback $k(\cdot)$ that is sought.

The approach as presented in $\S 1$ requires the Lyapunov function $V$ to be Lipschitz (in the zone under consideration). It is not known whether a globally asymptotically controllable system must admit a suitably Lipschitz $V$, but that is not quite the setting in which we work. Instead, Theorem 1 derives finite-time stabilizability, but to a close approximation of some inner level set $S(a)$, as a consequence of the supposed existence of a Lipschitz Lyapunov function. In contrast, [6] obtains asymptotic stabilizability to the origin (the case $S(a)=\{0\}$ ), and the proof uses Moreau-Yosida inf convolution to produce a Lipschitz Lyapunov function as an intermediate step. This methodology is also employed in [10], in a differential game setting. The direct use of a Lipschitz Lyapunov function, when it is possible, leads to a far more transparent feedback construction, and has the important consequence of yielding robustness, as we discuss presently. The fact that under mild assumptions, suitable Lipschitz functions exist leading to practical stabilization to any required tolerance, is proven in section 2.

Ledyaev and Sontag [21] have recently proved that there is a close relationship between the issues of "how regular a Lyapunov function does the system admit" and "how robust a stabilizing feedback does the system admit". Consider for example a perturbed equation $\dot{x}=f(x, k(x+p))$, where $p$ represents a measurement error. Full robustness of the feedback $k$ is taken to mean that for any $\epsilon$, there is a $\delta>0$ such that whenever the perturbation $p(t)$ satisfies $\|p(t)\| \leq \delta$ for all $t$, then stabilization to the $\epsilon$-ball takes place. This seems to have been studied by Hermes [17]. Then [21] asserts that the system admits a fully robust stabilizing feedback iff it admits a smooth $\left(C^{1}\right.$ or $\left.C^{\infty}\right)$ Lyapunov function. Thus the nonholonomic integrator, which can be stabilized by a discontinuous feedback (in view of [6]), does not admit a fully robust stabilizing feedback. It appears that Hermes [16] was the first to raise the question of robustness of discontinuous feedback with respect to measurement error.

The above concept of full robustness, unrelated as it is to the system sampling method that we employ, is not the one discussed in this article. Instead, we introduce a type of relative robustness in which we require the size of the measurement error to be limited as a function of the maximum step size $\delta$ of the underlying partition. This step size $\delta$ must still be small enough (for stabilization), but at the same time the individual steps must be big enough to preclude a possible chattering phenomenom, even in the presence of small errors. This consideration, which leads us to specify "reasonably uniform" sampling in section 3, appears to be new in this context. The term "reasonably uniform" is taken here to mean that the following hold :

$$
\frac{\delta}{2} \leq t_{i+1}-t_{i} \leq \delta \quad \forall i \geq 0,
$$

although it is possible to replace the factor $\frac{1}{2}$ by any constant in $(0,1)$. 
To conclude with the nonholonomic integrator, then, it turns out that the system does admit a stabilizing feedback to within any prescribed tolerance $r$, in the sense that we will have $\|x(t)\| \leq r$ for all $t \geq T$, whenever $x$ is a $\pi$-trajectory, where $\pi$ is a reasonably uniform partition whose diameter is sufficiently small, and whenever measurement and external error do not exceed a critical level related to the diameter (or sampling rate).

\section{A feedback construction}

For a given function $V: \mathbb{R}^{n} \longmapsto(-\infty, \infty]$, we shall deal frequently with the sublevel sets $S(r)$ defined as follows :

$$
S(r):=\left\{x \in \mathbb{R}^{n}: V(r) \leq r\right\} .
$$

In addition, the following sets are considered :

$$
S(a, b):=\left\{x \in \mathbb{R}^{n}: a \leq V(x) \leq b\right\} .
$$

Let $a$ and $b$ be two given numbers with $a<b$. The following hypotheses are made concerning the function $V$ and the system function $f$ :

(H1) $V$ is lower semicontinuous, $S(b) \neq \emptyset$, and for some $\eta>0, V$ is Lipschitz of rank $L_{V}$ on $S(a, b)+\eta B$ :

$$
|V(x)-V(y)| \leq L_{V}\|x-y\| \quad \forall(x, y) \in S(a, b)+\eta B .
$$

(H2) $\exists \delta_{1} \in(0, b-a)$ and $\delta_{2}>0$ such that

$$
S\left(a+\delta_{1}\right)+\delta_{2} B \subset S(b) .
$$

(H3) $f(x, u)$ is continuous on $S(b)+\eta B$ as a function of $x$ for each $u \in \mathcal{U}$, and $\exists m>0$ such that

$$
\| f(x, u \| \leq m \quad \forall x \in S(b)+\eta B, \forall u \in \mathcal{U} .
$$

(H4) $f$ is Lipschitz in $x$ of $\operatorname{rank} L_{f}$ on $S(a, b)+\eta B$ :

$$
\|f(x, u)-f(y, u)\| \leq L_{f}\|x-y\| \quad \forall x, y \in S(a, b)+\eta B, \forall u \in \mathcal{U} .
$$

(H5) There exists $\omega>0$ such that, for every $x \in S(a, b)+\eta B$, we have

$$
\inf _{v \in \operatorname{co} f(x, \mathcal{U})} D V(x ; v) \leq-\omega .
$$

Remark 1.1 We do not require that $f$ and $V$ be defined except on $S(b)+\eta B$; the Lipschitz conditions on these functions, as well as the Infinitesimal Decrease Condition (H5), are posited only on a neighborhood of $S(a, b)$. No hypotheses are made concerning the abstract set $\mathcal{U}$, nor on the nature of the dependence of $f$ on the control variable. 
Theorem 1 For any $\gamma>0$ sufficiently small, there exist positive numbers $\delta, T$ and a feedback $k: S(b)+\eta B \longmapsto \mathcal{U}$ such that whenever a partition $\pi$ satisfies $\operatorname{diam}(\pi)<\delta$, then any $\pi$-trajectory $x(\cdot)$ having $x(0) \in S(b)+\gamma B$ satisfies

$$
\begin{aligned}
& x(t) \in S(b)+\gamma B \quad \forall t \geq 0, \\
& x(t) \in S(a)+\gamma B \quad \forall t \geq T .
\end{aligned}
$$

Remark 1.2 Thus we almost recover the conclusion of the "ideal case" discussed in the Introduction, but in approximate terms, with a discontinuous feedback, and for a nonsmooth Lyapunov function satisfying localized hypotheses. The proof is constructive and gives estimates of $\gamma, \delta$ and $T$ in terms of the given data.

Remark 1.3 Note that (H5) is a weaker hypothesis than (3). An important fact used in the proof is that the Infinitesimal Decrease Condition (H5) has a strictly equivalent formulation in proximal terms, as follows :

$$
\inf _{u \in \mathcal{U}}\langle\zeta, f(x, u)\rangle \leq-\omega \quad \forall x \in S(a, b)+\eta B, \forall \zeta \in \partial_{P} V(x) .
$$

Here, $\partial_{P} V$ is the proximal subdifferential of $V$ [9]. The equivalence of the formulations is nontrivial, and is a consequence of Subbotin's Theorem [9, Theorem 3.4.2].

The proof of Theorem 1 is based upon defining a feedback control, via projections. The first two lemmas below guarantee that the projections lie in the set where the hypotheses are active.

Lemma 1.1 Let $\epsilon$ lie in $\left[0, \delta_{1}\right]$ and suppose that $x$ is a point in the set

$$
\left[S(a+\epsilon)+\min \left\{\delta_{2}, \eta\right\} B\right] \backslash S(a+\epsilon) .
$$

Then $x \in S(a, b)$, and if $s \in \operatorname{proj}(x, S(a+\epsilon))$, then $s \in S(a, b)+\eta B$.

Proof Since we have $S\left(a+\delta_{1}\right)+\delta_{2} B$ contained in $S(b)$ by hypothesis, it follows that $x$ lies in $S(b)$. Since $x$ does not belong to $S(a+\epsilon)$, we deduce $x \in S(a, b)$. Finally, we have

$$
\|s-x\|<\min \left\{\delta_{2}, \eta\right\} \leq \eta,
$$

whence $s \in S(a, b)+\eta B . \square$

Lemma 1.2 Let $0<\gamma<\eta / 2$, and suppose that for some $r^{\prime}$ and $r$ with $a \leq$ $r^{\prime}<r \leq b$ we have $x \in[S(r)+\gamma B] \backslash\left[S\left(r^{\prime}\right)+\gamma B\right]$. Then $x \in S(a, b)+\gamma B$, and if $s \in \operatorname{proj}(x, S(r))$, then $s \in S(a, b)+\eta B$.

Proof There exists $y \in S(r)$ having $\|y-x\|<\gamma$. Since $x$ does not belong to $S\left(r^{\prime}\right)+\gamma B$, we have $V(y)>r^{\prime}$ necessarily. Thus $y \in S(a, b)$ and $x \in S(a . b)+\gamma B$. Finally, we note $\|x-s\|<\gamma$, whence $\|y-s\|<2 \gamma$ and $s$ lies in $S(a, b)+\eta B$.

The next "solvability" result is central to our approach. The notation $u_{+}$ stands for $\max \{u, 0\}$. 
Lemma 1.3 For any $r \in[a, b]$, for any $x \in S(a, b)$, we have

$$
d(x, S(r)) \leq \frac{m}{\omega}(V(x)-r)_{+} .
$$

Proof We shall invoke results (and terminology) from [9] to give a short proof of this result, whose proof from first principles would be lengthy.

We define a lower semicontinuous function $g: \mathbb{R}^{n} \longmapsto[0, \infty]$ as follows :

$g(x):=(V(x)-r)_{+}+I_{S(b)}(x)$, where $I_{S(b)}(\cdot)$ is the indicator function of the set $S(b)$. At any point $x$ in the open set $C:=\{y: g(y)>0\}$ at which $g$ is finite, we have $x \in S(a, b)$, and the infinitesimal decrease condition implies that

$$
\inf \{D g(x ; v): v \in \operatorname{cof}(x, \mathcal{U})\} \leq-\omega .
$$

It follows from this that for any $\epsilon>0$, for any $x \in C$, for any $\zeta \in \partial_{P} g(x)$, there exists $u \in \mathcal{U}$ such that

$$
\langle\zeta, f(x, u)\rangle \leq-\omega+\epsilon .
$$

Since $\| f\left(x, u \| \leq m\right.$ and since $\epsilon>0$ is arbitrary, we derive $\|\zeta\| \geq \frac{\omega}{m}$. This verifies the hypothesis of the Solvability Theorem [9, Theorem 3.3.1] (applied with $V=\Omega=\mathbb{R}^{n}$ ), whose conclusion is precisely the desired one since $S(r)=$ $\{x: g(x)=0\}$.

We now proceed to fix $\gamma>0$ such that

$$
\gamma<\min \left\{\delta_{1}, \frac{\eta}{2}, \frac{\omega}{12 L_{f} L_{V}}\right\}
$$

and we define

$$
\beta:=\min \left\{\delta_{1}, \frac{(b-a)}{2}, \frac{\gamma \omega}{4 m}\right\} .
$$

Let $N$ be the first integer such that

$$
b-N \beta>a \geq b-(N+1) \beta .
$$

Note that $N \geq 1$ since $\beta<b-a$. We proceed to define certain sets $\Omega_{i}(i=$ $0,1, \cdots, N+1)$ that lie at the heart of our construction.

For $0 \leq i \leq N-1$, we set

$$
\Omega_{i}:=[S(b-i \beta)+\gamma B] \backslash[S(b-(i+1) \beta)+\gamma B] ;
$$

for $i=N$ we set

$$
\Omega_{N}:=[S(b-N \beta)+\gamma B] \backslash\left[S(b-N \beta)+\frac{\gamma}{4} B\right] ;
$$

and finally, we define $\Omega_{N+1}:=S(b-N \beta)+\frac{\gamma}{4} B$.

We now gather some facts about these sets.

\section{Lemma 1.4}


(a) The $\Omega_{i}$ are disjoint, and $\Omega_{i}$ is contained in $S(a, b)+\gamma B$ for $i \leq N$.

(b) $\bigcup_{i=0}^{N+1} \Omega_{i}=S(b)+\gamma B$.

(c) If $x \in \Omega_{i}$ for some $i \in\{0,1, \cdots, N\}$ and $s \in \operatorname{proj}(x, S(b-i \beta))$, then $s \in S(a, b)+\eta B$.

(d) $\left.S(b-i \beta)+\frac{\gamma}{4} B \subset S(b-(i+1) \beta)\right)+\gamma B \quad(i=0,1, \cdots, N-1)$.

(e) For every $i \in\{0,1, \cdots, N\}, \forall x \in \Omega_{i}$, we have $\frac{\gamma}{4} \leq d(x, S(b-i \beta))<\gamma$.

(f) $S(b-N \beta)+\frac{\gamma}{2} B \subset S(a)+\gamma B$.

\section{Proof}

(a): That the $\Omega_{i}$ are disjoint is evident; that they lie in $S(a, b)+\gamma B$ for $i \leq N$ follows from Lemma 1.2 for $i<N$ and from Lemma 1.1 for $i=N$ (recall that $b-N \beta-a \leq \beta \leq \delta_{1}$ and $\gamma<\delta_{2}$ ).

(b): Evident.

(c): Direct from Lemma $1.2(i<N)$ or Lemma $1.1(i=N)$.

(d): Let $x$ lie in $S(b-i \beta)+\frac{\gamma}{4} B$, and let $s \in S(b-i \beta)$ satisfy $\|x-s\|<\frac{\gamma}{4}$. Then $V(s) \leq b-i \beta$, and if $V(s) \leq b-(i+1) \beta$ the conclusion is immediate. Otherwise we have

$$
V(s)>b-(i+1) \beta>a,
$$

so that $s \in S(a, b)$. By lemma 1.3 there exists $y \in S(b-(i+1) \beta)$ such that

$$
\|s-y\| \leq \frac{m}{\omega}[V(s)-b+(i+1) \beta] \leq \frac{m \beta}{\omega} \leq \frac{\gamma}{2},
$$

in view of (7). Then $\|x-y\| \leq\|x-s\|+\|s-y\|<\frac{\gamma}{4}+\frac{\gamma}{2}<\gamma$, which established the desired conclusion.

(e): For $i=N$, this is immediate from the definition of $\Omega_{N}$; for $i<N$, it is a consequence of $(\mathrm{d})$.

(f): Let $x$ belong to $S(b-N \beta)+\frac{\gamma}{2} B$, and let $s \in S(b-N \beta)$ satisfy $\|x-s\|<\frac{\gamma}{2}$. If $V(s) \leq a$, then $x \in S(a)+\gamma B$. Otherwise, $s$ belongs to $S(a, b)$, and lemma 1.3 implies the existence of $y \in S(a)$ such that

$$
\|y-s\| \leq \frac{m}{\omega}[V(s)-a] \leq \frac{m}{\omega}[b-N \beta-a] \leq \frac{m \beta}{\omega} \leq \frac{\gamma}{2} .
$$

But then $\|x-y\|<\gamma$, so again $x \in S(a)+\gamma B$.

Lemma 1.5 Let $x \in \Omega_{i}(i=0,1, \cdots, N)$, and let $s \in \operatorname{proj}(x, S(b-i \beta))$. Then there exists $u \in \mathcal{U}$ such that

$$
\langle x-s, f(s, u)\rangle \leq \frac{-\omega}{2 L_{V}}\|x-s\| .
$$


Proof By definition, $x-s$ lies in the proximal normal cone $N^{P}(x, S(b-i \beta))$. Note that $s$ lies in $S(a, b)+\eta B$ (by Lemma 1.2 for $i<N$, Lemma 1.1 for $i=N$ ), so that $V$ is Lipschitz of rank $L_{V}$ in a neighborhood of $s$. A basic calculus result $[9,1.11 .26]$ yields the existence of $\lambda>0$ such that $\lambda(x-s) \in \partial_{L} V(s)$, and necessarily $\lambda\|x-s\| \leq L_{V}$. In accord with Remark 1.1, there exists $u \in \mathcal{U}$ such that

$$
\langle\lambda(x-s), F(s, u)\rangle \leq \frac{-\omega}{2} .
$$

The result follows.

\section{Defining the feedback}

We now define a feedback $k(\cdot)$ on $S(b)+\gamma B$ as follows. If $x \in \Omega_{i}$ for some $i \in\{0,1, \cdots, N\}$, then we set $k(x)=u$, where $u$ corresponds to $x$ (and a projection $s$ ) as in lemma 1.5. There remain the points $x$ in $\Omega_{N+1}$ to consider (see Lemma 1.4 (b)). For such $x$, we define $k(x)$ to be any point in $\mathcal{U}$, for example one which minimizes $\|f(x, u)\|$.

The remainder of the proof consists in establishing that for suitably small mesh size, any $\pi$-trajectory generated by $k(\cdot)$ with initial condition in $S(b)+\gamma B$ remains in $S(b)+\gamma B$, enters $S(a)+\gamma B$ within a certain (uniform) time, and then remains in that set subsequently.

We consider countable partitions $\left\{t_{j}\right\}$ such that $t_{0}=0, \lim _{j \rightarrow \infty} t_{j}=\infty$, and such that $t_{j+1}-t_{j} \leq \delta \quad \forall j \geq 0$, where $\delta$ is any positive number satisfying

$$
\delta<\min \left\{\frac{\gamma}{4 m}, \frac{\omega}{6 m L_{f} L_{V}}, \frac{\gamma \omega}{48 m^{2} L_{V}}, 1\right\} .
$$

For such a partition, let $x_{0}$ be any point in $S(b)+\gamma B$, and let $x(\cdot)$ be a $\pi$ trajectory with $x(0)=x_{0}$. We denote $x\left(t_{j}\right)$ by $x_{j}$, and we set $\Delta:=\frac{\omega}{60 L_{V}}$.

Lemma 1.6 For some $t_{j} \in \pi$, suppose that $x_{j} \in \Omega_{i}, i \in\{0,1, \cdots, N\}$. Then $x(t) \in S(b)+\gamma B$ for $t \in\left[t_{j}, t_{j+1}\right]$, and

$$
d(x(t), S(b-i \beta)) \leq d\left(x_{j}, S(b-i \beta)\right)-\Delta\left(t-t_{j}\right) \quad \forall t \in\left[t_{j}, t_{j+1}\right] .
$$

Proof We have $x_{j} \in S(a, b)+\gamma B$ by Lemma 1.4 (a), and $\|\dot{x}(t)\| \leq m$ while $x(t)$ lies in $S(b)+\eta B$. Since $\delta m<\frac{\gamma}{4}$ by $(8)$ and $\gamma<\frac{\eta}{2}$, it follows that $x(t)$ lies in $S(a, b)+\eta B$ for $t \in\left[t_{j}, t_{j+1}\right]$, as does the point s that figures in the definition of $k\left(x_{j}\right)$; this was pointed out in the proof of Lemma 1.5, where we also deduced the inequality

$$
\left\langle x_{j}-s, f\left(s, k\left(x_{j}\right)\right)\right\rangle \leq \frac{-\omega}{2 L_{V}}\left\|x_{j}-s\right\| .
$$

We fix $t \in\left(t_{j}, t_{j+1}\right)$ and set $\Psi:=\frac{x(t)-s}{\|x(t)-s\|}$. (Note that $x(t) \neq s$, since $\left\|x_{j}-s\right\| \geq \frac{\gamma}{4}$ by Lemma $1.4(\mathrm{e})$, and since $\left\|x(t)-x_{j}\right\|<\delta m<\frac{\gamma}{4}$.)

We now observe two inequalities :

$$
d(x(t), S(b-i \beta)) \leq\|x(t)-s\|=\langle\Psi, x(t)-s\rangle,
$$




$$
d\left(x_{j}, S(b-i \beta)\right)=\left\|x_{j}-s\right\| \geq\left\langle\Psi, x_{j}-s\right\rangle .
$$

These together imply

$$
\begin{aligned}
d(x(t), S(b-i \beta))-d\left(x_{j}, S(b-i \beta)\right) & \leq\left\langle\Psi, x(t)-x_{j}\right\rangle \\
& =\tau\left\langle\Psi, f_{j}\right\rangle,
\end{aligned}
$$

where we introduce the notation $\tau:=t-t_{j}$,

$$
x(t)=x_{j}+\tau f_{j}, f_{j}:=\frac{1}{\tau} \int_{t_{j}}^{t} f\left(x(r), k\left(x_{j}\right)\right) d r .
$$

We also set

$$
\hat{f}_{j}:=f\left(s, k\left(x_{j}\right)\right)=\frac{1}{\tau} \int_{t_{j}}^{t} f\left(s, k\left(x_{j}\right)\right) d r .
$$

Note that $\left\|f_{j}-\hat{f}_{j}\right\| \leq \frac{1}{\tau} \int_{t_{j}}^{t} L_{f}\|x(r)-s\| d r$

(the Lipschitz condition applies because we are in $S(a, b)+\eta B$ )

$$
\begin{aligned}
& \leq \frac{1}{\tau} \int_{t_{j}}^{t} L_{f}\left(\left\|x(r)-x_{j}\right\|+\left\|x_{j}-s\right\|\right) d r \\
& \leq L_{f}\left(\tau m+d\left(x_{j}, S(b-i \beta)\right)\right) .
\end{aligned}
$$

It follows from this and (9) that we have

$$
\begin{aligned}
\left\langle x_{j}-s, f_{j}\right\rangle= & \left\langle x_{j}-s, \hat{f}_{j}+f_{j}-f_{j}\right\rangle \\
\leq & \frac{-\omega}{2 L_{V}}\left\|x_{j}-s\right\|+L_{f} d\left(x_{j}, S(b-i \beta)\right)\left\{\tau m+d\left(x_{j}, S(b-i \beta)\right)\right\} \\
\leq & d\left(x_{j}, S(b-i \beta)\right)\left[\frac{-\omega}{2 L_{V}}+L_{f} \delta m+\gamma L_{f}\right] \\
& \left(\text { since } \tau<\delta \text { and } d\left(x_{j}, S(b-i \beta)\right) \leq \gamma\right) \\
\leq & d\left(x_{j}, S(b-i \beta)\right)\left[\frac{-\omega}{2 L_{V}}+\frac{\omega}{6 L_{V}}+\frac{\omega}{6 L_{V}}\right] \\
& (\text { we have invoked }(8) \text { and }(6)) \\
\leq & -\frac{\gamma \omega}{24 L_{V}} \\
& \quad\left(\operatorname{since} d\left(x_{j}, S(b-i \beta)\right) \geq \frac{\gamma}{4}\right. \text { by Lemma 1.4 (e)). }
\end{aligned}
$$

We shall use this bound on $\left\langle x_{j}-s, f_{j}\right\rangle$ to derive one on $\left\langle x(t)-s, f_{j}\right\rangle$ as follows:

$$
\begin{aligned}
\left\langle x(t)-s, f_{j}\right\rangle & =\left\langle x_{j}+\tau f_{j}-s, f_{j}\right\rangle \leq\left\langle x_{j}-s, f_{j}\right\rangle+\tau\left\|f_{j}\right\|^{2} \\
& \leq \frac{-\gamma \omega}{24 L_{V}}+\delta m^{2} \leq \frac{-\gamma \omega}{48 L_{V}}
\end{aligned}
$$

(in light of (8)).

We also have

$$
\begin{aligned}
\|x(t)-s\|=\left\|x_{j}+\tau f_{j}-s\right\| & \leq\left\|x_{j}-s\right\|+\tau\left\|f_{j}\right\| \leq \gamma+\delta m \\
& <\frac{5 \gamma}{4}(\text { by }(8)) .
\end{aligned}
$$


Combining this with (11) we arrive at

$$
\left\langle\Psi, f_{j}\right\rangle=\left\langle\frac{x(t)-s}{\|x(t)-s\|}, f_{j}\right\rangle \leq \frac{-\gamma \omega}{48 L_{V}} / \frac{5 \gamma}{4}=-\Delta .
$$

Together with (10), this gives the inequality asserted by the Lemma. Since this inequality evidently implies

$$
d(x(t), S(b-i \beta))<\gamma,
$$

it also follows that $x(t) \in S(b)+\gamma B$.

Lemma 1.7 If $x_{j} \in \Omega_{i}$ where $0 \leq i \leq N$, then $x_{j+1}$ lies in $\Omega_{i^{\prime}}$ for some $i^{\prime} \geq i$.

Proof Since $x_{j} \in \Omega_{i}$, we have $d\left(x_{j}, S(b-i \beta)\right)<\gamma$, and (by Lemma 1.6) $d\left(x_{j+1}, S(b-i \beta)\right)<\gamma$. Now let $1 \leq k<j$. Since $S(b-i \beta) \subset S(b-(k+1) \beta)$, we deduce $d\left(x_{j+1}, S(b-(k+1) \beta)\right)<\gamma$. But then $x_{j+1} \notin \Omega_{k}$ by definition of $\Omega_{k}$. Since $x_{j+1} \in S(b)+\gamma B$ by Lemma 1.6 , we must have $x_{j+1} \in \Omega_{i^{\prime}}$ for some $i^{\prime} \geq i$, in view of Lemma $1.4(\mathrm{~b})$

Lemma 1.8 If $x(\tau) \in \Omega_{N+1}$ for some $\tau \in \pi$, then $x(t) \in S(a)+\gamma B \quad \forall t \geq \tau$.

Proof We know that $x(\tau)$ lies in the interior of $S(a)+\gamma B$ by Lemma 1.4 (f). For $t>\tau$, as long as $d(x(t), S(b-N \beta))$ does not attain or exceed $\frac{\gamma}{2}$, then $x(t)$ remains in $S(a)+\gamma B$. Thus $\|\dot{x}(t)\|$ remains bounded by $m$ and no blow-up occurs (i.e., $x(t)$ is well-defined).

It suffices therefore to prove that the continuous function $g(t):=d(x(t), S(b-$ $N \beta)$ ) does not become greater than or equal to $\frac{\gamma}{2}$ for some $t_{0}>\tau$. We have $g(\tau) \leq \frac{\gamma}{4}$.

Note that $S(b-N \beta)+\gamma B$ lies in $S(b)+\gamma B$, so that the bound $\|\dot{x}\| \leq m$ continues to apply for some time after any positive point $t_{0}$ at which $g(t)$ becomes equal to $\frac{\gamma}{2}$. Thus $g(t)$ is locally well-defined. Yet Lemma 1.6 (for $i=N$ ) shows that $g$ is decreasing when $g(t)$ lies between $\frac{\gamma}{4}$ and $\gamma$. The conclusion is that $g(t) \leq \frac{\gamma}{2} \quad \forall t>\tau\left(\right.$ or in fact that $g(t)$ never even exceeds $\left.\frac{\gamma}{4}\right)$

One last lemma and the proof is complete.

Lemma 1.9 Let $T:=\left(1+\frac{b-a}{\beta}\right)\left(1+\frac{45 \gamma L_{V}}{\omega}\right)$. Then

$$
x(t) \in S(a)+\gamma B \quad \forall t \geq T .
$$

Proof In view of Lemma 1.8 and Lemma 1.4 (f), it suffices to prove that there is a node $\tau \in \pi$ with $\tau \leq T$ for which $x(\tau) \in \Omega_{N+1}$. Note that $x(0)$ belongs to some $\Omega_{i}(0 \leq i \leq N+1)$ by Lemma 1.4 (b); if $i=N+1$ we are done, so assume $i \leq N$. Since $\delta<1$ by (8), there is a node $\tau_{1}$ lying in the open interval $(\sigma, \sigma+1)$, where $\sigma:=\frac{3 \gamma}{4 \Delta}$. By Lemma 1.7, $x\left(\tau_{1}\right)$ belongs either to $\Omega_{i}$ or to $\Omega_{i^{\prime}}$ for some $i^{\prime}>i$. In the former case, it follows that $x(t)$ lies in $\Omega_{i}$ for every node $t \in \pi$ lying between 0 and $\tau_{1}$, and the inequality of Lemma 1.6 applies to give

$$
\begin{aligned}
d\left(x\left(\tau_{1}\right), S(b-i \beta)\right) & \leq d(x(0), S(b-i \beta))-\Delta \tau_{1} \\
& <\gamma-\frac{3 \gamma}{4}=\frac{\gamma}{4} .
\end{aligned}
$$


However, the left side is no less than $\frac{\gamma}{4}$ by Lemma 1.4 (e). This contradiction shows that, in fact, $x\left(\tau_{1}\right)$ must belong to some $\Omega_{i^{\prime}}$ for an index $i^{\prime}>i$. The same argument, beginning now at $\left(\tau_{1}, x\left(\tau_{1}\right)\right)$, yields the existence of a node $\tau_{2} \in \pi$ with $\tau_{2} \leq 2 \sigma+2$ such that $x\left(\tau_{2}\right)$ belongs to $\Omega_{i^{\prime \prime}}$, where $i^{\prime \prime}>i^{\prime}$. Continuing in this manner, we find that (since there are at most $N+1$ steps as above prior to landing in $\left.\Omega_{N+1}\right)$, there is a node $\tau \in \pi$ with $\tau \leq(N+1)(\sigma+1)$ such that $x(\tau) \in \Omega_{N+1}$. But $N<\frac{b-a}{\beta}$ implies that the $T$ defined in the statement of the Lemma is greater than $(N+1)(\sigma+1)$.

\section{Construction of a Lyapunov function}

We show in this section thst under reasonable assumptions, there always exist Lyapunov functions having the properties required for the feedback construction of the preceding section, and giving rise to practical feedback stabilization of arbitrarily prescribed range. While the result below seems new and the approach to proving it has some novel features, there is a familiar heuristic at work : the Lyapunov function is contructed as the value function associated with a parametrized family of optimal control problems.

The function $f(x, u)$ describing the dynamics is supposed in this section to satisfy much the same regularity conditions as before. Specifically, we require that for any bounded subset $S$ of $\mathbb{R}^{n}$, there exist constants $m=m(S)$ and $L=L(S)$ such that

$$
\begin{gathered}
\|f(x, u)\| \leq m \quad \forall x \in S, \forall u \in \mathcal{U} \\
\|f(x, u)-f(y, u)\| \leq L\|x-y\| \quad \forall x, y \in S, \forall u \in \mathcal{U} .
\end{gathered}
$$

(As before, $\mathcal{U}$ is just an abstract set, and no hypotheses are made concerning the nature of the dependence of $f$ on $u$.)

In addition, we require controllability to the ball of radius $r_{0}$ via relaxed trajectories. Let us now proceed to make this precise. We are given $r_{0} \geq 0$, and we define a multifunction $\Gamma$ on $\mathbb{R}^{n}$ by

$$
\Gamma(x):=\operatorname{clco}\{f(x, u): u \in \mathcal{U}\} .
$$

By "trajectory" we mean an absolutely continuous function $x(\cdot)$ on an interval $[0, T]$ such that

$$
\dot{x}(t) \in \Gamma(x(t)) \quad \text { a.e. } \quad t \in[0, T] .
$$

Given $\alpha \in \mathbb{R}^{n}$, we define $T_{0}(\cdot)$ as the least time required for a trajectory to go from $\alpha$ to the closed ball $r_{0} \bar{B}$ :

$$
T_{0}(\alpha):=\inf \left\{T \geq 0: x(\cdot) \text { is a trajectory on }[0, T], x(0)=\alpha,\|x(T)\| \leq r_{0}\right\} .
$$

the controllability hypothesis that we make is that every $\alpha$ admits a trajectory steering it to $r_{0} \bar{B}$ in finite time, a time which goes to zero as $\alpha$ approaches $r_{0} \bar{B}$. Equivalently :

$$
T_{0}(\alpha)<\infty \quad \forall \alpha \in \mathbb{R}^{n} \text {, and } \lim _{\|\alpha\| \downarrow r_{0}} T_{0}(\alpha)=0
$$


Theorem 2 For any pair $r$ and $R$ with $R>r>r_{0}$, there exist a function $V$ and numbers $a, b, \gamma, \eta$ such that

$$
S(a)+\gamma B \subset B(0, r) \subset \bar{B}(0, R) \subset S(b)+\gamma B,
$$

and such that $V$ satisfies all the hypotheses of Theorem 1; in addition $S(b)$ is compact. Thus there is a feedback defined on a neighborhood of $\bar{B}(0, R)$ which stabilizes the state to $B(0, r)$ in the sense of Theorem 1.

We begin by defining another multifunction $\tilde{\Gamma}$ (more useful than $\Gamma$ for being compact-valued) :

$$
\tilde{\Gamma}(x):=\operatorname{clco}\left\{\frac{v}{1+\|v\|}: v \in \Gamma(x)\right\} .
$$

We set

$\tilde{T}_{0}(\alpha):=\inf \left\{T \geq 0: x(\cdot)\right.$ is a $\tilde{\Gamma}-$ trajectory on $\left.[0, T], x(0)=\alpha,\|x(T)\| \leq r_{0}\right\}$.

Evidently (or by convention) we have $\tilde{T}_{0}=0$ on $r_{0} \bar{B}$.

Lemma 2.1 (a) $\tilde{\Gamma}$ is locally Lipschitz and has nonempty convex compact values in $\bar{B}(0,1)$.

(b) $\tilde{T}_{0}(\alpha)$ is finite $\forall \alpha \in \mathbb{R}^{n}$.

(c) $\lim _{\|\alpha\| \downarrow r_{0}} \tilde{T}_{0}(\alpha)=0$.

(d) There exists a positive number $\epsilon$ such that whenever $\alpha \in B\left(0, r_{0}+\epsilon\right)$, and whenever the $\tilde{\Gamma}$-trajectory $x(\cdot)$ has $x(0)=\alpha$ and $\|x(T)\| \leq r_{0}$ for some $T \leq \tilde{T}_{0}(\alpha)+\epsilon$, then we have $\|x\|_{\infty} \leq r_{0}+1$. We can suppose $\epsilon<1, \epsilon<r-r_{0}$, and that

$$
\sup \left\{\tilde{T}_{0}(\alpha): \alpha \in B\left(0, r_{0}+\epsilon\right)\right\}<\frac{\left(r-r_{0}\right)^{2}}{17\left(r_{0}+1\right)} .
$$

Proof We omit the routine proof of $(a)$. For $(b)$, let $\alpha \in \mathbb{R}^{n}$ be given. By assumption, there is a $\Gamma$-trajectory $x$ on an interval $[0, T]$ such that $x(0)=$ $\alpha,\|x(T)\|=r_{0}$. We set

$$
\tilde{T}:=\int_{0}^{T}(1+\|\dot{x}(t)\|) d t
$$

and we define a function $\tilde{x}$ on $[0, \tilde{T}]$ by

$$
\tilde{x}(\tau):=x(t),
$$

where $t=t(\tau)$ is determined in $[0, T]$ by

$$
\tau=\int_{0}^{t}(1+\|\dot{x}(r)\|) d r
$$


(this change of variables or time scale is known as the Erdmann Transform.) Then

$$
\frac{d \tilde{x}}{d \tau}=\frac{\dot{x}(t)}{1+\|\dot{x}(t)\|} \in \tilde{\Gamma}(\tilde{x}(\tau)) \quad \text { a.e. }
$$

so that $\tilde{x}$ is a $\tilde{\Gamma}$-trajectory. Hence $\tilde{T}_{0}(\alpha) \leq \tilde{T}<\infty$.

We turn now to (c). Let $\alpha_{i}$ be a sequence for which $\left\|\alpha_{i}\right\|$ decreases to $r_{0}$. Then $T_{0}\left(\alpha_{i}\right) \rightarrow 0$ by assumption. Let $m$ be such that $\|f(x, u)\| \leq m$ for $(x, u) \in \bar{B}\left(0, r_{0}+1\right) \times \mathcal{U}$. Then, as soon as $T_{0}\left(\alpha_{i}\right)$ is strictly less than $\frac{\overline{1}}{m}$, there is a $\Gamma$-trajectory $x_{i}$ on an interval $\left[0, T_{i}\right]$ such that

$$
x_{i}(0)=\alpha_{i},\left\|x_{i}\left(T_{i}\right)\right\| \leq r_{0}, T_{i}<\frac{1}{m}, T_{i}<T_{0}\left(\alpha_{i}\right)+\frac{1}{i} .
$$

It follows that $\left\|x_{i}\right\|_{\infty}<r_{0}+1$. Now let $\tilde{x}_{i}$ be the Erdmann Transform of $x_{i}$ as given above. Then

$$
\tilde{T}_{0}\left(\alpha_{i}\right) \leq \tilde{T}_{i}=\int_{0}^{T_{i}}\left(1+\left\|\dot{x}_{i}(t)\right\|\right) d t \leq(1+m) T_{i}<(1+m)\left(T_{0}\left(\alpha_{i}\right)+\frac{1}{i}\right) .
$$

It follows that $\tilde{T}_{0}\left(\alpha_{i}\right) \rightarrow 0$, as required.

We now examine $(\mathrm{d})$. If the assertion is false, there exist a sequence $\alpha_{i}$ with $\left\|\alpha_{i}\right\| \downarrow r_{0}$ and corresponding $\tilde{\Gamma}$-trajectories $x_{i}$ with $x_{i}(0)=\alpha_{i},\left\|x_{i}\left(T_{i}\right)\right\| \leq r_{0}$, such that

$$
T_{i} \leq \tilde{T}_{0}\left(\alpha_{i}\right)+\frac{1}{i},\left\|x_{i}\right\|_{\infty}>r_{0}+1
$$

Since $\tilde{T}_{0}\left(\alpha_{i}\right) \rightarrow 0$ by (c), we have $T_{i} \rightarrow 0$. On the other hand, there is a subinterval of $\left[0, T_{i}\right]$ in which $\left\|x_{i}\right\|$ goes from being $r_{0}+1$ to $r_{0}$, and since $\left\|\dot{x}_{i}(t)\right\| \leq 1$ the length of that subinterval (and hence, $T_{i}$ ) is at least 1 . This contradiction establishes the first part of $(d)$; the rest follows immediatly by shrinking $\epsilon$ as required, in light of (c)

We proceed now to define a new multifunction $F(x)$ whose effect is to enlarge the set $\tilde{\Gamma}(x)$ for $\|x\|<r_{0}+\epsilon$. We set

$$
F(x):= \begin{cases}\tilde{\Gamma}(x) & \text { for }\|x\| \geq r_{0}+\epsilon \\ \tilde{\Gamma}(x)+2\left[\frac{r_{0}+\epsilon-\|x\|}{\epsilon}\right] \bar{B} & \text { for } r_{0} \leq\|x\| \leq r_{0}+\epsilon \\ \tilde{\Gamma}(x)+2 \bar{B} & \text { for }\|x\| \leq r_{0}\end{cases}
$$

Having done this, we define a value function $V(\cdot)$ on $\mathbb{R}^{n}$ in terms of the trajectories of $F$ as follows :

$$
V(\alpha):=\inf \left\{\int_{0}^{T}\|x(t)\| d t: T \geq 0, x(0)=\alpha, \dot{x} \in F(x) a . e .,\|x(T)\| \leq r_{0}\right\} .
$$

We stress that $T$ is a choice variable here, in this free time problem. 
Lemma 2.2 (a) $F$ is compact and convex-valued, uniformly bounded and locally Lipschitz.

(b) $V(\cdot)$ is finite-valued and lower semicontinuous, and the infinum defining $V(\alpha)$ is attained for every $\alpha$.

(c) $V(\alpha)>0$ iff $\|\alpha\|>r_{0}$, and $\lim _{\|\alpha\| \downarrow r_{0}} V(\alpha)=0$.

(d) The sublevel sets $S(b):=\{x: V(x) \leq b\}$ of $V$ are compact.

Proof The assertions of (a) are immediate. Since $F(x)$ is uniformly bounded, the attainment and the lower semicontinuity asserted in (b) follow from standard "compactness of trajectories" arguments; see [9, Chapter 4] for details. The first assertion of (c) is clear, and the other one stems from Lemma 2.1 as follows.

Let $\alpha \in B\left(0, r_{0}+\epsilon\right)$, and let the $\tilde{\Gamma}$-trajectory $x$ satisfy $x(0)=\alpha,\|x(T)\| \leq r_{0}$, and $T \leq \tilde{T}_{0}(\alpha)+\delta$, for some $\delta \in(0, \epsilon)$.

Then $\|x\|_{\infty} \leq r_{0}+1$, and we deduce

$$
V(\alpha) \leq \int_{0}^{T}\|x(t)\| d t \leq\left(\tilde{T}_{0}(\alpha)+\delta\right)\left(r_{0}+1\right) .
$$

Since $\tilde{T}_{0}(\alpha) \downarrow 0$ as $\|\alpha\| \downarrow r_{0}$, (c) follows. Finally we turn to (d). If $\|\alpha\|>r_{0}+1+\epsilon$, then the time required for a trajectory to go from $\|x\|=\alpha$ to $\|x\|=r_{0}+\epsilon$ is at least $\alpha-r_{0}-\epsilon$. But then $V(\alpha) \geq\left(r_{0}+\epsilon\right)\left(\|\alpha\|-r_{0}-\epsilon\right)$. This established (d)

The next step invokes Hamiltonian conditions for optimal control, and uses the lower Hamiltonian $h$ associated with $F$ :

$$
h(x, p):=\min \{\langle p, v\rangle: v \in F(x)\} .
$$

Lemma 2.3 Let $\zeta \in \partial_{P} V(\alpha)$, where $\|\alpha\|>r_{0}$. Let $x$ be a trajectory solving the problem that defines $V(\alpha)$, with associated time $T$. Then there exists an absolutely continuous function $p$ on $[0, T]$ such that

$$
\begin{aligned}
\left(-\dot{p}-\frac{x}{\|x\|}, \dot{x}\right) & \in \partial_{C} h(x, p) \quad \text { a.e. } \quad t \in[0, T] \\
p(0) & =\zeta \\
h(x(t), p(t))+\|x(t)\| & =0 \quad \forall t \in[0, T] .
\end{aligned}
$$

Proof By definition of $\partial_{P} V(\alpha)$, we have for some $\sigma \geq 0$ and for all $\alpha^{\prime}$ near $\alpha$,

$$
V\left(\alpha^{\prime}\right)+\sigma\left\|\alpha^{\prime}-\alpha\right\|^{2}-\left\langle\zeta, \alpha^{\prime}\right\rangle \geq-\langle\zeta, \alpha\rangle .
$$

Let $x^{\prime}$ be a trajectory near $x$ (in the $L^{\infty}$ norm), put $\alpha^{\prime}=x^{\prime}(0)$ and $\alpha=x(0)$ and rearrange to derive that $x^{\prime}(\cdot)=x(\cdot)$ solves locally the problem of minimizing

$$
\int_{0}^{T^{\prime}}\left\|x^{\prime}(t)\right\| d t-\left\langle\zeta, x^{\prime}(0)\right\rangle+\sigma\left\|x^{\prime}(0)-x(0)\right\|^{2}
$$


over the trajectories $x^{\prime}$ for $F$ satisfying $\left\|x^{\prime}\left(T^{\prime}\right)\right\| \leq r_{0}$. (Here $T^{\prime}$ and $x^{\prime}(0)$ are free.) We apply the corollary of Theorem 3.6.1 of [5] (in reverse time) to deduce the existence of an absolutely continuous function $q$ on $[0, T]$ satisfying

$$
\begin{aligned}
(-\dot{q}, \dot{x}) & \in \partial_{C}[H(x, q)-\|x\|](x, q) \quad \text { a.e. } \quad t \in[0, T], \\
q(0) & =-\zeta \\
H(x(t), q(t))-\|x(t)\| & =0, \quad t \in[0, T]
\end{aligned}
$$

where $H(x, p)$ is the function $-h(x,-p)$ and $\partial_{C}$ denotes the generalized gradient. The Hamiltonian inclusion above implies

$$
\left(\dot{q}-\frac{x}{\|x\|}, \dot{x}\right) \in \partial_{C} h(x,-q) \quad \text { a.e. }
$$

Now putting $p:=-q$ gives the conclusions the form asserted in the statement of the lemma.

Lemma 2.4 For any constant $c>0$, there is a constant $M_{c}$ with the following property. If $\alpha \in S(c)$ and if the trajectory $x$ on $[0, T]$ attains the infimum defining $V(\alpha)$, then $\|x\|_{\infty} \leq M_{c}, T \leq M_{c}$.

Proof If $\|x\|_{\infty}>c+r_{0}+1$, then the time required for $\|x\|$ to attain the value $r_{0}+1$ exceeds $c$ (since $\left.\|\dot{x}\| \leq 1\right)$. But then $V(\alpha) \geq\left(1+r_{0}\right) c>c$. This shows that $\|x\|_{\infty}$ is bounded by $c+r_{0}+1$. By Lemma $2.1(\mathrm{c}), \lim _{\|\alpha\| \downarrow r_{0}} \tilde{T}_{0}(\alpha)=0$. So there exists $\rho>r_{0}$ such that $\forall x \in B(0, \rho),\left|\tilde{T}_{0}(x)\right| \leq 1$. Now for $\|\alpha\| \geq \rho$, let $\tau_{\rho}$ denote the first time that $\|x(t)\|$ attains the value $\rho$. Then $V(\alpha) \geq \rho \tau_{\rho}$, whence $\tau_{\rho} \leq \frac{c}{\rho}$ for $\alpha \in S(c)$.

We deduce that

$$
\begin{aligned}
\tilde{T}_{0}(\alpha) & \leq \tau_{\rho}+1 \quad(\text { by construction of } \rho) \\
& \leq \frac{c}{\rho}+1 .
\end{aligned}
$$

If $\|\alpha\|<\rho$, the same bound evidently holds.

It suffices now to set

$$
M_{c}:=\max \left\{\frac{c}{\rho}+1, c+r_{0}+1\right\}
$$

Lemma 2.5 $V$ is locally Lipschitz on $\mathbb{R}^{n}$.

Proof We prove first that $V$ is locally Lipschitz on the open set $\{V>0\}=$ $\operatorname{comp}\left(\bar{B}\left(0, r_{0}\right)\right)$. Let $\alpha_{0}$ belong to this set, take any $\delta>0$ such that $\delta<\left\|\alpha_{0}\right\|-r_{0}$, and any element $\zeta \in \partial_{P} V(\alpha)$, where

$$
\left\|\alpha-\alpha_{0}\right\|<\delta, V(\alpha) \leq V\left(\alpha_{0}\right)+\delta=: c .
$$


The conclusions of Lemma 2.4 are available for any trajectory solving the $V(\alpha)$ problem. If $K$ is a Lipschitz constant for $F$ on the ball $B\left(0, M_{c}+1\right.$ ) (where $M_{c}$ comes from Lemma 2.4), then the Hamiltonian inclusion (13) implies

$$
\|\dot{p}\| \leq K\|p\|+1
$$

The condition (15) at $t=T$ gives $-\|p(T)\| \geq-r_{0}$ since $\|x(T)\|=r_{0}$, and since $F(x(T))=\operatorname{clcof}(x(T), \mathcal{U})+2 \bar{B} \supset \bar{B}$. Thus $\|p(T)\| \leq r_{0}$. This, together with (20) and Gronwall's Lemma, leads to

$$
\begin{aligned}
\|\zeta\|=\|p(0)\| & \leq e^{K T}\|p(T)\|+\int_{0}^{T} e^{K(T-s)} d s \\
& \leq r_{0} e^{K T}+\frac{e^{K T}-1}{K} \\
& \leq r_{0} e^{K M_{c}}+\frac{e^{K M_{c}}-1}{K},
\end{aligned}
$$

since $T \leq M_{c}$ by Lemma 2.4. This establishes a uniform bound on elements of $\partial_{P} V(\alpha)$ whenever $\alpha$ satisfies (19), which proves that $V$ is Lipschitz on a neighborhood of $\alpha_{0}$ [9, 1.11.11]. Thus $V$ is locally Lipschitz on the set where it is strictly positive.

There is a neighborhood $N$ of $\bar{B}\left(0, r_{0}\right)$ on which $V$ is bounded above, in view of Lemma 2.2(c). The argument above therefore yields a bound $L$ on elements of $\partial_{P} V(\alpha)$ for all $\alpha \in N \backslash \bar{B}\left(0, r_{0}\right)$, so that $V$ is uniformly Lipschitz of rank $L$ on $\alpha \in N \backslash \bar{B}\left(0, r_{0}\right)$ by [9, Theorem 1.7.3]. Of course, $V=0$ on $\bar{B}\left(0, r_{0}\right)$. That $V$ is Lipschitz on $N$, and hence locally Lipschitz on $\mathbb{R}^{n}$, now follows. $\square$

\section{Lemma 2.6}

$$
\sup \left\{V(\alpha): \alpha \in B\left(0, r_{0}+\epsilon\right)\right\}<\inf \left\{V(\alpha):\|\alpha\| \geq \frac{r_{1}-r_{0}}{2}\right\}
$$

Proof Let $\|\alpha\|<r_{0}+\epsilon$, fix $\delta \in(0, \epsilon)$ and let the trajectory $x$ on $[0, T]$ satisfy $x(0)=\alpha,\|x(T)\| \leq r_{0}, T<\tilde{T}_{0}(\alpha)+\delta$. Then by Lemma 2.1 we have $\|x\|_{\infty} \leq r_{0}+1$ and so

$$
V(\alpha) \leq \int_{0}^{T}\|x(t)\| d t \leq\left(\tilde{T}_{0}(\alpha)+\delta\right)\left(r_{0}+1\right) .
$$

We derive $V(\alpha) \leq\left(r_{0}+1\right) \tilde{T}_{0}(\alpha)$, and (from (12))

$$
V(\alpha)<\frac{\left(r-r_{0}\right)^{2}}{17}
$$

Now let $\|\alpha\| \geq \frac{r-r_{0}}{2}$, and let $x$ solve the problem defining $V(\alpha)$. There is an interval of length at least $\frac{r-r_{0}}{4}$ during which $\|x(t)\| \geq \frac{r-r_{0}}{4}$ (since $\|\dot{x}\| \leq 1$ ), whence

$$
V(\alpha)>\frac{\left(r-r_{0}\right)^{2}}{16} .
$$

The result follows. $\square$ 
Lemma 2.7 There exist positive numbers $a, b, \eta$ with $a<b$ such that

$$
S(a)+\eta B \subset B(0, r) \subset \bar{B}(0, R) \subset S(b)
$$

and

$$
S(a, b)+\eta B \subset\left\{\alpha: r_{0}+\epsilon<\|\alpha\|\right\} .
$$

Proof Pick a number $a>0$ lying between the two quantities in the statement of Lemma 2.6. Then evidently

$$
S(a) \subset B\left(0, \frac{r-r_{0}}{2}\right), \quad \operatorname{cl}(\operatorname{comp} S(a)) \subset \operatorname{comp} \bar{B}\left(0, r_{0}+\epsilon\right),
$$

whence $S(a)+\eta B \subset B(0, r)$ for $\eta>0$ suitable small. It also follows that the compact set $\operatorname{cl} S(a, b)$ is contained in the open set $\left\{\alpha: r_{0}+\epsilon<\|\alpha\|\right\}$. By shrinking $\eta$ further if necessary, this will be true of $\operatorname{cl} S(a, b)+\eta B$ as well. Finally, any $b$ suitably large will satisfy $\bar{B}(0, R) \subset S(b)$, since $V$ is bounded on bounded sets.

Lemma 2.8 The Infinitesimal Decrease Condition (H5) of $\S$ holds on $S(a, b)+$ $\eta B$, with $\omega:=-r_{0}-\epsilon$.

Proof As remarked in $\S 1$, it suffices to show that for any $\alpha \in S(a, b)+\eta B$, for any $\zeta \in \partial_{P} V(\alpha)$, one has :

$$
\inf \{\langle\zeta, f(\alpha, u)\rangle: u \in \mathcal{U}\} \leq-r_{0}-\epsilon .
$$

Let $x$ be a trajectory solving the problem defining $V(\alpha)$.

Then, by Lemma 2.3, we have (at $t=0$ ) :

$$
h(\alpha, \zeta)+\|\alpha\|=0 .
$$

Since $\|\alpha\|>r_{0}+\epsilon$ by Lemma 2.7 , we have

$$
F(\alpha)=\tilde{\Gamma}(\alpha),
$$

so that the preceding equality yields, for any $\delta>0$, the existence of some element $v \in \Gamma(\alpha)$ such that

$$
\left\langle\zeta, \frac{v}{1+\|v\|}\right\rangle \leq-\|\alpha\|+\delta<-\left(r_{0}+\epsilon\right)+\delta .
$$

For $\delta$ small enough, the right side is negative, whence

$$
\langle\zeta, v\rangle<-\left(r_{0}+\epsilon\right)+\delta .
$$

Given that $\Gamma(x):=\operatorname{clco} f(x, \mathcal{U})$, this yields the existence of $u \in \mathcal{U}$ for which

$$
\langle\zeta, f(\alpha, u)\rangle<-\left(r_{0}+\epsilon\right)+2 \delta .
$$

Since $\delta$ is arbitrarily small, (21) ensues.

Since $S(b)$ is compact, $f$ is Lipschitz in $x$ and bounded on $S(b)+\eta B$, in accord with hypotheses (H3) (H4) of section 1. When the level sets are compact, (H2) always holds. The verification of this is the last property to confirm. 
Lemma 2.9 Hypothesis (H2) holds.

Proof If (H2) fails, then there exist sequences $\alpha_{i} \in \mathbb{R}^{n}, \epsilon_{i} \downarrow 0$, and $u_{i} \in B(0,1)$ such that

$$
V\left(\alpha_{i}\right) \leq a+\epsilon_{i} \text { and } V\left(\alpha_{i}+u_{i}\right)>b .
$$

Since $S(b)$ is compact, we can suppose by passing to a subsequence that $\alpha_{i} \rightarrow \alpha_{0}$. Then $V\left(\alpha_{0}\right) \geq b>a \geq V\left(\alpha_{0}\right)$, a contradiction.

The setting of Theorem 1 is established, and Theorem 2 is proved.

\section{Robustness}

We prove in this section that the feedback constructed in $\S 1$ is robust with respect to small measurement error and persistent external disturbance, in a precise sense that requires two stipulations. The first is that the measurement error must not exceed in order of magnitude the step size of the underlying discretization, a condition which appears to be rather natural. The second requirement is perhaps more surprising, and surfaces from the nature of the feedback construction. It dictates that each step be "big enough" (while continuing to be "small enough") so as to counteract the measurement error by means of the attractive effect inherent in the construction. Thus the partitions used to discretize the effect of the control are taken to be "reasonably uniform".

Our perturbed system is modeled by

$$
\dot{x}=f(x, k(x+p))+q,
$$

where the external disturbance $q:[0, \infty) \longmapsto \mathbb{R}^{n}$ is a bounded measurable function :

$$
\|q(t)\| \leq E_{q} \quad t \geq 0 \quad \text { a.e. }
$$

Given a partition $\pi=\left\{t_{i}\right\}_{i>0}$ of $[0, \infty)$ and the initial condition $x_{0}$, the resulting $\pi$-trajectory of our perturbed system is defined by successively solving the differential equation

$$
\dot{x}(t)=f\left(x(t), k\left(x\left(t_{i}\right)+p_{i}\right)\right)+q(t), \quad t \in\left[t_{i}, t_{i+1}\right],
$$

with $x(0)=x_{0}$. The continuous function $x(t)$ is the real state of the system, while the sequence $\left\{x\left(t_{i}\right)+p_{i}\right\}$ corresponds to the inexact measurements used to select control values.

Theorem 3 The feedback $k: S(b)+\gamma B \rightarrow \mathcal{U}$ constructed in Theorem 1 is robust in the following sense. There exist positive numbers $\delta_{0}, T$ and $E_{q}$ such that, for every $\delta \in\left(0, \delta_{0}\right)$ there exists $E_{p}(\delta)>0$ having the following property: for any partition $\pi=\left\{t_{i}\right\}_{i \geq 0}$ having

$$
\frac{\delta}{2} \leq t_{i+1}-t_{i} \leq \delta \quad i \geq 0,
$$


where $0<\delta<\delta_{0}$, for any set of measurement errors $\left\{p_{i}\right\}_{i \geq 0}$ having

$$
\left\|p_{i}\right\| \leq E_{p} \quad i \geq 0
$$

for any initial condition $x_{0}$ such that $x_{0}+p_{0} \in S(b)+\gamma B$, for any disturbance $q$ having $\|q\|_{\infty} \leq E_{q}$, the resulting $\pi$-trajectory $x$ satisfies

$$
\begin{gathered}
x\left(t_{i}\right)+p_{i} \in S(b)+\gamma B \quad \forall i \geq 0, \\
x(t) \in S(b)+2 \gamma B \quad \forall t \geq 0, \\
x(t) \in S(a)+\gamma B \quad \forall t \geq T .
\end{gathered}
$$

Remarks 3.1 (a) Note that unlike $T$ and $E_{q}$, the maximum admissible measurement error $E_{p}$ depends on $\delta$. Note also that (in contrast to Theorem 1) $x(t)$ may not lie in $S(b)+\gamma B$ for all $t$, although for large it must do so. (For example, we do not require $x(0) \in S(b)+\gamma B$.)

However, the "observed values" of the state, namely the values $x\left(t_{i}\right)+$ $p_{i}(i \geq 0)$, all fall in $S(b)+\gamma B$ the domain of definition of $k$.

(b) Certain other kinds of error, for example a disturbance $d(\cdot)$ entering into the dynamics in the form $\dot{x}=f(x, k(x)+d)$, can be reduced to that of external disturbance by positing suitable continuity of $f$ in the control variable.

(c) The maximum admissible disturbance measure $E_{q}$ will be seen to be proportional to $\frac{\omega}{L_{V}}$. This has a natural physical meaning, as can easily be seen in the case of smooth $V$ and a continuous feedback $k(x)$ such that $\langle\nabla V(x), f(x, k(x))\rangle \leq-W(x)$.

Then we see that the perturbed system

$$
\dot{x}=f(x, k(x))+q
$$

is stabilized by $k$ if $\|q\|_{\infty}<\frac{W(x)}{\|\nabla V(x)\|}$ for every $x$, a bound akin to that involving $\frac{\omega}{L_{V}}$.

We adapt the proof of Theorem 1, whose first five lemmas hold with no change whatever, as does the definition of $k(\cdot)$. Recall that $\gamma, \beta$ and $N$ were introduced (earlier; see (6) (7)). We now define our upper bound for $\delta$ :

$$
\begin{aligned}
\delta_{0} & :=\min \left\{\frac{6 \gamma L_{V}}{\omega}, 1, \frac{\gamma \omega}{24 L_{V}\left(m+\frac{\omega}{6 L_{V}}+1\right)^{2}}, \frac{\gamma}{20 m}\right\} \\
E_{q} & :=\frac{\omega}{6 L_{V}} \\
T & :=\left(1+\frac{b-a}{\beta}\right)\left(1+\frac{81 \gamma L_{V}}{\omega}\right)
\end{aligned}
$$


(this $T$ differs slightly from the one in Theorem 1) and we let $\pi=\left\{t_{i}\right\}_{i \geq 0}$ be a partition as described $\mathrm{i}$ the statement of Theorem 3, with corresponding measurement errors $\left\{p_{i}\right\}_{i \geq 0}$ having $\left\|p_{i}\right\| \leq E_{p}$ for some $E_{p}>0$ satisfying

$$
E_{p}<\min \left\{\frac{3 \gamma}{80}, \delta, \frac{\delta \omega}{432 L_{V}}\right\}
$$

We also admit any disturbance $q(\cdot)$ for which $\|q\|_{\infty} \leq E_{q}$, and we take $x_{0}$ such that $x_{0}+p_{0} \in S(b)+\gamma B$. We shall show that the corresponding $\pi$-trajectory has the required properties. We introduce the notation

$$
x_{i}:=x\left(t_{i}\right), y_{i}:=x_{i}+p_{i}
$$

for the actual and the measures space state values at time $t_{i}$, and proceed to develop modified versions of the fourlast lemmas figuring in the proof of Theorem 1. We set $\tilde{\Delta}:=\frac{\omega}{108 L_{V}}$.

Lemma 3.1 For some $t_{j} \in \pi$, suppose that $y_{j} \in \Omega_{i}, i \in\{0,1, \cdots, N\}$. Then

$$
\begin{gathered}
x(t) \in S(b)+2 \gamma B \subset S(b)+\eta B, \quad t_{j} \leq t \leq t_{j+1} \\
y_{j} \in S(b)+\gamma B, y_{j+1} \in S(b)+\gamma B, \\
d\left(y_{j+1}, S(b-i \beta)\right) \leq d\left(y_{j}, S(b-i \beta)\right)-\tilde{\Delta}\left(t_{j+1}-t_{j}\right) .
\end{gathered}
$$

Proof Note that $y_{j} \in S(b)+\gamma B$ by Lemma 1.4; it will follow from the last conclusion of the current Lemma that $y_{j+1} \in S(b)+\gamma B$. Also, $\left\|x_{j}-y_{j}\right\|=$ $\left\|p_{j}\right\| \leq E_{p}$, together with $\left\|x(t)-x_{j}\right\| \leq \delta m$, yield

$$
x(t) \in S(a, b)+\gamma B+\left(E_{p}+\delta m\right) B \subset S(b)+2 \gamma B,
$$

since $E_{p}+\delta m<\gamma$ in view of (22) and (25). Since $2 \gamma<\eta$ by (6), this gives $x(t) \in S(b)+\eta B$. By Lemma 1.5 we have

$$
\left\langle y_{j}-s, f\left(s, k\left(y_{j}\right)\right)\right\rangle \leq-\frac{\omega}{2 L_{V}}\left\|y_{j}-s\right\|
$$

where $s \in \operatorname{proj}\left(y_{j}, S(b-i \beta)\right)$. Fix $t \in\left(t_{j}, t_{j+1}\right)$ and set

$$
\Psi:=\frac{x(t)-s}{\|x(t)-s\|} .
$$

Note that $x(t) \neq s$ since $\left\|y_{j}-s\right\| \geq \frac{\gamma}{4}$ by Lemma $1.4(\mathrm{e})$, while

$$
\left\|y_{j}-x(t)\right\| \leq\left\|y_{j}-x_{j}\right\|+\left\|x_{j}-x(t)\right\|<E_{p}+\delta m<\frac{\gamma}{4},
$$

as already noted.

We observe the relations

$$
\begin{aligned}
d(x(t), S(b-i \beta)) & \leq\|x(t)-s\|=\langle\Psi, x(t)-s\rangle, \\
d\left(y_{j}, S(b-i \beta)\right) & =\left\|y_{j}-s\right\| \geq\left\langle\Psi, y_{j}-s\right\rangle,
\end{aligned}
$$


whence

$$
\begin{aligned}
d(x(t), S(b-i \beta))-d\left(y_{j}, S(b-i \beta)\right) & \leq\left\langle\Psi, x(t)-y_{j}\right\rangle \\
& =\left\langle\Psi, x_{j}+\tau\left(f_{j}+q_{j}\right)-y_{j}\right\rangle \\
& \leq \tau\left\langle\Psi, f_{j}+q_{j}\right\rangle+\left\|p_{j}\right\|
\end{aligned}
$$

where we have introduced

$$
\begin{aligned}
\tau & :=t-t_{j} \\
f_{j} & :=\frac{1}{\tau} \int_{t_{j}}^{t} f\left(x(r), k\left(y_{j}\right)\right) d r \\
q_{j} & :=\frac{1}{\tau} \int_{t_{j}}^{t} q(r) d r .
\end{aligned}
$$

We also set $\hat{f}_{j}:=f\left(s, k\left(y_{j}\right)\right)$; note $\left\|\hat{f}_{j}\right\| \leq m$. We have

$$
\begin{aligned}
\left\|f_{j}-\hat{f}_{j}\right\| & \leq \frac{1}{\tau} \int_{t_{j}}^{t} L_{f}\|x(r)-s\| d r \leq \frac{L_{f}}{\tau} \int_{t_{j}}^{t}\left(\left\|x(r)-x_{j}\right\|+\left\|x_{j}-s\right\|\right) d r \\
& \leq L_{f}\left(\delta m+\left\|x_{j}-y_{j}\right\|+\left\|y_{j}-s\right\|\right) \\
& \leq L_{f}\left(\delta m+E_{p}+\gamma\right) \leq \frac{5}{4} L_{f} \gamma
\end{aligned}
$$

We deduce

$$
\begin{aligned}
\left\langle x(t)-s, f_{j}+q_{j}\right\rangle & =\left\langle x_{j}+\tau\left(f_{j}+q_{j}\right)-s, f_{j}+q_{j}\right\rangle \\
& =\left\langle y_{j}-s-p_{j}, f_{j}+q_{j}\right\rangle+\tau\left\|f_{j}+q_{j}\right\|^{2} \\
\leq & \left\langle y_{j}-s, f_{j}\right\rangle+E_{p}\left(m+E_{q}\right)+\delta\left(m+E_{q}\right)^{2} \\
& =\left\langle y_{j}-s, \hat{f}_{j}\right\rangle+\left\langle y_{j}-s, f_{j}-\hat{f}_{j}\right\rangle+\left(m+E_{q}\right)\left[\delta m+\delta E_{q}+E_{p}\right] \\
\leq & -\frac{\omega}{2 L_{V}}\left\|y_{j}-s\right\|+\frac{5}{4}\left\|y_{j}-s\right\| L_{f} \gamma+\left(m+E_{q}\right)\left[\delta m+\delta E_{q}+E_{p}\right] \\
& \quad(\text { where we have used }(26) \text { and }(28)) \\
\leq & d\left(y_{j}, S(b-i \beta)\right)\left[-\frac{\omega}{2 L_{V}}+\frac{\omega}{6 L_{V}}\right]+\delta\left[m+E_{q}+1\right]^{2} \\
& \quad\left(\text { by }(6), \text { and since } E_{p}<\delta \text { by }(25)\right) \\
\leq & \frac{\gamma}{4}\left[-\frac{\omega}{3 L_{V}}\right]+\frac{\gamma \omega}{24 L_{V}}=-\frac{\gamma \omega}{24 L_{V}}
\end{aligned}
$$

(since $d\left(y_{j}, S(b-i \beta)\right) \geq \frac{\gamma}{4}$ by Lemma 1.4, and since $\delta<\delta_{0}$ defined by (22)). Note also that

$$
\begin{aligned}
\|x(t)-s\| & =\left\|y_{j}-p_{j}+\tau\left(f_{j}+q_{j}\right)-s\right\| \\
& \leq \gamma+E_{p}+\delta\left(m+E_{q}\right) \leq \frac{5 \gamma}{4}+\delta E_{q}<\frac{9 \gamma}{4}
\end{aligned}
$$


(note $\delta E_{q}<\gamma$ because of $\delta<\delta_{0}$, in view of (22) (23)).

It follows that

$$
\left\langle\Psi, f_{j}+q_{j}\right\rangle=\left\langle\frac{x(t)-s}{\|x(t)-s\|}, f_{j}+q_{j}\right\rangle \leq-\frac{(\gamma \omega) /\left(24 L_{V}\right)}{(9 \gamma) / 4}=-\frac{\omega}{54 L_{V}} .
$$

Substituting into (27) leads to

$$
d(x(t), S(b-i \beta))-d\left(y_{j}, S(b-i \beta)\right) \leq-2 \tilde{\Delta}\left(t-t_{j}\right)+E_{p} .
$$

We obtain from this

$$
\begin{aligned}
d\left(y_{j+1}, S(b-i \beta)\right)-d\left(y_{j}, S(b-i \beta)\right) & \leq\left\|y_{j+1}-x\left(t_{j+1}\right)\right\|-2 \tilde{\Delta}\left(t-t_{j}\right)+E_{p} \\
& \leq-\tilde{\Delta}\left(t_{j+1}-t_{j}\right)+\left[2 E_{p}-\tilde{\Delta}\left(t_{j+1}-t_{j}\right)\right] \\
& \leq-\tilde{\Delta}\left(t_{j+1}-t_{j}\right),
\end{aligned}
$$

by $(25)$, and since $t_{j+1}-t_{j} \geq \frac{\delta}{2}$.

Lemma 3.2 If $y_{j} \in \Omega_{i}$, where $i \leq N$, then $y_{j+1}$ lies in $\Omega_{k}$ for some $k \geq i$.

Proof We know that $y_{j+1} \in \Omega_{k}$ for some $k$, since $y_{j+1}$ belongs to $S(b)+\gamma B$ by Lemma 3.1. Suppose that $k<i$. We have $d\left(y_{j}, S(b-i \beta)\right)<\gamma$ by definition of $\Omega_{i}$, and Lemma 3.1 implies $d\left(y_{j+1}, S(b-i \beta)\right)<\gamma$. But $S(b-i \beta) \subset S(b-(k+1) \beta)$, so that $d\left(y_{j+1}, S(b-(k+1) \beta)\right)<\gamma$. But then $y_{j+1} \notin \Omega_{k}$ by definition of $\Omega_{k}$ (note that $k \leq N$ ). This contradiction proves the Lemma. $\square$

Lemma 3.3 If $\tau \in \pi$ is such that $y(\tau) \in \Omega_{N+1}$, then

$$
x(t) \in S(a)+\gamma B \quad \forall t \geq \tau .
$$

Proof We first establish

$$
d\left(y\left(\tau^{\prime}\right), S(b-N \beta)\right) \leq \frac{2 \gamma}{5} \text { for all nodes } \tau^{\prime} \geq \tau .
$$

We consider first $\tau^{\prime}=\tau+1$. We have $d(y(\tau), S(b-N \beta)) \leq \frac{\gamma}{4}$, whence

$$
\begin{aligned}
d(y(\tau+1), S(b-N \beta)) & \leq d(x(\tau+1), S(b-N \beta))+E_{p} \\
& \leq d(x(\tau), S(b-N \beta))+E_{p}+m \delta \\
& \leq d(y(\tau), S(b-N \beta))+2 E_{p}+m \delta \\
& <\frac{\gamma}{4}+\frac{3 \gamma}{20}=\frac{2 \gamma}{5}(\text { by }(25)) .
\end{aligned}
$$

If $d(y(\tau+1), S(b-N \beta))$ is in fact $\leq \frac{\gamma}{4}$, then this same argument yields

$$
d(y(\tau+2), S(b-N \beta)) \leq \frac{2 \gamma}{5} .
$$

If however $d(y(\tau+1), S(b-N \beta))>\frac{\gamma}{4}$, then $y(\tau+1)$ lies in $\Omega_{N}$ by definition, and Lemma 3.1 yields again $d(y(\tau+2), S(b-N \beta))<\frac{2 \gamma}{5}$. Continuing in this 
way, we obtain (29) for all nodes $\tau^{\prime} \geq \tau$.

We use (29) to argue as follows : let $t \geq \tau$, and let $\tau^{\prime} \geq \tau$ be a node adjacent to $t$; then

$$
\begin{aligned}
d(x(t), S(b-N \beta)) & \leq d\left(x\left(\tau^{\prime}\right), S(b-N \beta)\right)+\delta m \\
& \leq d\left(y\left(\tau^{\prime}\right), S(b-N \beta)\right)+E_{p}+\delta m \\
& <\frac{2 \gamma}{5}+\frac{\gamma}{10}=\frac{\gamma}{2}(\text { by }(25)) .
\end{aligned}
$$

This gives $x(t) \in S(a)+\gamma B$ by Lemma 1.4 (f)

Lemma 3.4 Let

$$
T:=\left(1+\frac{b-a}{\beta}\right)\left(1+\frac{81 \gamma L_{V}}{\omega}\right) .
$$

Then $x(t) \in S(a)+\gamma B \quad \forall t \geq T$.

Proof In view of Lemma 3.3, it suffices to prove that some node $\tau \in \pi$ with $\tau \leq T$ is such that $y(\tau) \in \Omega_{N+1}$. The argument is identical to that used to prove Lemma 1.8 , with $\Delta$ replaced by $\tilde{\Delta}$, and applied to the $y_{i}$ rather than the $x_{i}$

\section{References}

[1] Z. Arstein. Stabilization with relaxed controls. Nonlinear Analysis TMA, 7:1163-1173, 1983.

[2] A. Bacciotti. Local Stabilizability of Nonlinear Control Systems. World Scientific, 1992.

[3] A. Bacciotti and L. Rosier. Lyapunov and Lagrange stability : inverse theorems for discontinuous systems. Math. Control Signals Systems, 11:101128, 1998.

[4] R.W. Brockett. Asymptotic stability and feedback stabilization. In Birkhäuser, editor, Differential Geometric Control Theory, pages 181-191. R.W. Brockett, R.S. Millman, and H.J. Sussmann, eds., 1983.

[5] F.H. Clarke. Optimization and Nonsmooth Analysis. Wiley-Interscience, New-York, 1983.

[6] F.H. Clarke, Yu.S. Ledyaev, E.D. Sontag, and A.I. Subbotin. Asymptotic Controllability Implies Feedback Stabilization. I.E.E.E. Trans. Aut. Control, 42:1394-1407, 1997.

[7] F.H. Clarke, Yu.S. Ledyaev, and R.J. Stern. Asymptotic stability and smooth Lyapunov functions. J. DIfferential Equations, 149:69-114, 1998. 
[8] F.H. Clarke, Yu.S. Ledyaev, R.J. Stern, and P.R. Wolenski. Qualitatives properties of trajectories of control systems: a survey. J. Dyn. Control Sys., 1:1-48, 1995.

[9] F.H. Clarke, Yu.S. Ledyaev, R.J. Stern, and P.R. Wolenski. Nonsmooth Analysis and Control Theory. Springer-Verlag, Graduate Texts in Mathematics, 1998.

[10] F.H. Clarke, Yu.S. Ledyaev, and A.I. Subbotin. The synthesis of universal pursuit strategies in differential games. SIAM J. Control and Optim., 35:552-561, 1997.

[11] J-M. Coron. Global asymptotic stabilization for controllable systems without drift. Math. Cont. Sig. Sys., 5:295-312, 1992.

[12] J-M. Coron and L. Rosier. A relation between continuous time-varying and discontinuous feedback stabilization. J. Math. Syst., Estimation, Control, 4:67-84, 1994.

[13] J.M. Coron, L. Praly, and A. Teel. Feedback stabilization of nonlinear systems : Sufficients conditions and Lyapunov and input-output techniques. In A. Isidori, editor, Trends in Control. Springer-Verlag, New-York, 1995.

[14] A.F. Filippov. Differential Equations with Discontinuous Righthand Sides. Kluwer Academic Publishers, 1988.

[15] R. Freeman and P.V. Kokotovic. Robust Nonlinear Control Design. StateSpace and Lyapounov Techniques. Birkhäuser, 1996.

[16] H. Hermes. Discontinuous vector fields and feedback control. In J.K. Hale and J.P. LaSalle, editors, Differential Equations and Dynamic Systems. Academic Press, New-York, 1967.

[17] H. Hermes. Resonance, stabilizing feedback controls, and regularity of viscosity solutions of Hamilton-Jacobi-Bellman equations. Math. Control Signals Systems, 9:59-72, 1996.

[18] A. Isidori. Nonlinear Control Systems. Springer-Verlag, 1995. 3rd Edition.

[19] N.N. Krasovskii and A.I. Subbotin. Game-Theoretical Control Problems. Springer-Verlag, New-York, 1988.

[20] V. Lakshmikantham, S. Leela, and A. Martynyuk. Practical Stability of Nonlinear Systems. World Scientific, Singapore, 1990.

[21] Yu.S. Ledyaev and E.D. Sontag. A lyapunov characterization of robust stabilization. To appear.

[22] G. Leitmann. One approach to the control of uncertain dynamical systems. Appl. Math. Comput., 70:261-272, 1995. 
[23] Y. Lin, E.D. Sontag, and Y. Wang. A smooth converse Lyapounov theorem for robust stability. SIAM J. Control and Optim., 34:124-160, 1996.

[24] E.P. Ryan. On brockett's condition for smooth stabilizability and its necessity in a context of nonsmooth feedback. S.I.A.M. J. Control Optim., 32:1597-1604, 1994.

[25] E.D. Sontag. A lyapunov-like characterization of asymptotic controllability. SIAM J. Control and Optim., 21:462-471, 1983.

[26] E.D. Sontag. Mathematical Control Theory. Springer-Verlag, New-York, 1990. (Second Edition, 1998).

[27] E.D. Sontag and H.J. Sussmann. Nonsmooth control-Lyapunov functions. In Proc. I.E.E.E. Conf. Decision and Control, New Orleans, pages 61-81, Boston, 1990. Birkhäuser.

[28] E.D. Sontag and H.J. Sussmann. Remarks on continuous feedback. In Proc. I.E.E.E. Conf. Decision and Control, Albuquerque, pages 916-921, Piscataway, December 1980. IEEE Publications.

[29] A.I. Subbotin. Generalized Solutions of First-Order PDEs. Birkhäuser, Boston, 1995.

[30] J. Tsinias. A lyapunov description of stability in control systems. Nonlinear Analysis TMA, 13:3-74, 1989. 